\title{
Information and Small Group Effectiveness in Large Quasilinear Economies
}

\author{
Yusuke Kamishiro* Roberto Serrano ${ }^{\dagger}$ and Myrna Wooders ${ }^{\ddagger}$
}

This version: October 2015

\begin{abstract}
In large quasilinear economies, we provide sufficient conditions to establish the nonemptiness of several versions of approximate interim cores with endogenous communication. This is done by proving nonemptiness of approximate ex post cores satisfying incentive compatibility. When the number of agents with private information remains finite, the main argument relies on an adaptation of the small group effectiveness condition, previously proposed for games of complete information. The result goes through when all agents are informationally small.

Keywords: Large Quasilinear Economies; Approximate Cores; Small Group Effectiveness; Asymmetric Information.

JEL Classification: C71, C72, D51, D82.
\end{abstract}

\footnotetext{
${ }^{*}$ Dept. of Economics, Kanto Gakuin University; ykamishi@kanto-gakuin.ac.jp

${ }^{\dagger}$ Dept. of Economics, Brown University; roberto_serrano@brown.edu

${ }^{\ddagger}$ Dept. of Economics, Vanderbilt University; myrna.wooders@vanderbilt.edu
} 


\section{Introduction}

This paper takes up the issue of nonemptiness of the core in interim economies with asymmetric information. Although Wilson (1978) proved nonemptiness of the largest possible core -the coarse core- in convex exchange economies, he also showed that the smallest possible core - the fine core- can easily be empty. The finding is very different as soon as incentive constraints are incorporated, in the sense that even the incentive compatible coarse core may be empty (Vohra, 1999), even in well-behaved domains such as quasilinear economies (Forges, Mertens, and Vohra, 2002). All these results concern cores with exogenous communication.

The current study also restricts attention to quasilinear preferences and focuses on large economies. In cases in which incentive constraints can be dropped, it first argues that cores with endogenous communication are nonempty. More important, when incentive constraints are still nontrivial, it adapts results from complete information games, such as Wooders (1994), which rely on a condition of small group effectiveness (SGE), in order to prove nonemptiness of approximate cores. SGE dictates that all or almost all gains to coalition formation can be realized by partitions of the total player set into relatively small coalitions. SGE holds when there is a small number of informed agents and an unbounded number of uninformed agents; a particular case of interest is what we shall call in Section 3 monopolists of scarce information. On the other hand, when there are many agents of each of a finite number of types, as in Section 4, SGE is equivalent to the apparently mild condition of boundedness of average -per capita- payoffs (Wooders, 1994, Theorem 4).

While extending the SGE condition to incomplete information settings is challenging, because details about the information structure will in general 
matter, we take an indirect route that is sufficient for our purposes. Indeed, we rely on the SGE condition stated for ex post economies, and on the results of Dutta and Vohra (2005) and Kamishiro and Serrano (2011). These previous results establish that having an ex post core allocation that is incentive compatible is sufficient in quasilinear economies to show that the smallest possible core with endogenous communication be nonempty. ${ }^{1}$

Thus, this paper focuses mainly on ex post sequences of economies derived from economic data for economies with a finite number of types of agents. These sequences are constructed in such a way that all agents of each type possess exactly the same information. The sequences can be constructed so that there are only a few - even only one - copies of some agents. In particular, we consider sequences in which only one agent is informed and possesses information not available elsewhere in the economy. We establish a nonemptiness of approximate interim cores result when the number of (uninformed) agents becomes sufficiently large. This is done through establishing nonemptiness of approximate ex post cores satisfying incentive compatibility and then appealing to the results described in the previous paragraph. Roughly, the main ideas of the result are that, since in any state of the economy the core is nonempty, given $\varepsilon>0$ from SGE it holds that if the number of uninformed agents is sufficiently large, then each agent with no information can be "taxed" $\varepsilon$ and an agent with scarce information paid an amount $\varepsilon$ times the number of uninformed agents. Thus, the informed agent is promised a sufficiently large payoff, whatever the state of the world, so that he has no incentive to withhold information.

Finally, as argued in Section 4, our main result extends to large economies in which all agents are informationally small in the sense of McLean and

\footnotetext{
${ }^{1}$ This core is referred to in Kamishiro and Serrano (2011) as the core with respect to equilibrium blocking of unrestricted random communication mechanisms.
} 
Postlewaite (2002), but the reason is different. In such settings, SGE also holds because there are no 'scarce types', that is, there are many copies of each agent. The nontrivial step of establishing the existence of an ex post core allocations satisfying incentive compatibility relies on a $\varepsilon$-transfer to a residual set of agents who receive signals that are uninformative about the true state of the world. With such transfers ensured, the inclusion of such ex post core allocations in the interim cores with endogenous communication completes the argument, and gives us nonemptiness of any such approximate interim core. $^{2}$

This is the plan of the paper. Section 2 presents the model. Section 3 focuses on the main result, based on a negligible number of informed agents. Section 4 considers sequences of economies in which all agents are informationally small. Section 5 concludes.

\section{The Model}

We shall consider a model with many agents whose characteristics are chosen from a finite set of types. However, in the presence of asymmetric information, we need to be careful about the distribution of information across agents in order to define sequences of economies. We shall first assume that two copies of the same type have exactly the same information. ${ }^{3}$ With this type of sequence, incentive constraints are absent when more than one copy of each agent is present. Thus, either agents have zero informational size when there are multiple copies of each informed agent, or they are informational monopolists otherwise. We shall also consider in the sequel (Section

\footnotetext{
${ }^{2}$ We are able to show this result for replica sequences; the case of more general sequences seems more challenging.

${ }^{3}$ In the literature of core convergence with asymmetric information, this sequence is called ex post replicas; see Serrano, Vohra and Volij (2001).
} 
4) different sequences of economies, in which agent copies' private signals on the true state of nature are independent. In such a model, agents may be informationally small, but incentive constraints are nonvacuous. ${ }^{4}$

\subsection{Pre-Economies and Economies}

Let $N=\{1,2, \ldots, j, \ldots, n\}$ denote a fixed finite set of agent types. Let $I(\subset N)$ denote a subset of $N$ consisting of types of agents with private information. For $j \in I$ we use $T_{j}$ to denote a set of possible pieces of private information $t_{j}$ for an agent of type $j$ concerning the state of the world. We will use the notation $t_{-j}$ to denote $\left(t_{j^{\prime}}\right)_{j^{\prime} \neq j}$. Similarly $T_{-j}=\prod_{j^{\prime} \neq j} T_{j^{\prime}}$. Let $T:=\prod_{j \in I} T_{j}$ denote the set of possible states of the world. Obviously, $N \backslash I$ is the set of agent types in $N$ with no private information. ${ }^{5}$

Let $e_{j} \in \mathbb{R}_{+}^{L}$ denote the (state-independent) endowment of an agent of type $j \in N$. For each state $t \in T$ and for each $j \in N$ let $u_{j}\left(x_{j}, t\right)=$ $v_{j}\left(x_{j}^{-L}, t\right)+x_{j}^{L}$ denote the utility function of an agent of type $j$. Note that $u_{j}\left(x_{j}, t\right)$ is quasilinear with the $L^{t h}$ good as numeraire. We also assume that $v_{j}$ is concave.

We say that $\left(N, I,\left(T_{j}, u_{j}\right)_{j=1, \ldots, n}\right)$ comprises a pre-economy. With the specification of a number of agents of each type, the pre-economy determines an economy.

A profile on $N$ is a function $f$ from $N$ to $Z_{+}$, the nonnegative integers: we interpret $f(j)$ as the number of agents of the same type (that is, identical in preferences, endowment and information). Note that a profile $f$ describes a set of agents consisting of $f(j)$ agents of type $j$ for each $j$ and thus determines

\footnotetext{
${ }^{4}$ This replication process was first considered in McLean and Postlewaite (2002).

${ }^{5}$ Defining the set of states as the product over finite sets of agent types is convenient. The dimensionality of the state space does not increase, even after increasing the number of agents, as we do in large economies; see, e.g., Serrano, Vohra, and Volij (2001).
} 
an economy. Given a profile $f$, a sub-profile of $f$ is a profile $f^{\prime}$ satisfying $f^{\prime} \leq f$. Let $\|f\|$ denote the number of agents in a group represented by a profile $f$, i.e., $\|f\|=\sum_{j \in N} f(j)$. Given the set of types $N$ and a profile $f$, define the agent set as

$$
\mathbf{N}=\{(i, j): j=1, \ldots, n ; i=1, \ldots, f(j)\}
$$

To save on notation, we abuse language slightly and refer to agent $(i, j)$ as agent $i$ of type $j$.

Given a profile $f$ and a state of the world $t$ define

$$
\Psi(f, t)=\max \sum_{j} f(j) u_{j}\left(x_{j}, t\right) \quad \text { subject to } \quad \sum_{j} f(j)\left[e_{j}-x_{j}\right]=0 .
$$

The function $\Psi$, mapping (in a given state) profiles into maximal total utility subject to the feasibility constraint, determines a pre-game, a pair $(N, \Psi)$, where $N$ is a set of player types and $\Psi$ is a worth function, specifying the total utility achievable by any group of agents. The pair $(N, \Psi)$ is the pregame determined by the pre-economy $\left(N, I,\left(T_{j}, u_{j}\right)_{j=1, \ldots, n}\right)$. Note that in deriving the pre-game we have taken into account that, from concavity of utility functions, the maximal total utility achievable by any set of agents, using their own resources, is achieved by an allocation of commodities that treats all agents of the same type equally. Also, this total utility can be achieved by the group consisting of all agents in the set; that is, we could take the maximum over all partitions of the set of agents but this would not yield a different result.

\subsection{Interim Economies and Core Notions}

We assume that agents have a common prior probability distribution $q$ defined on $T$, and that no type is redundant, i.e., $q\left(t_{j}\right)>0$ for all $t_{j} \in T_{j}$ for 
all $j \in N$. At the interim stage, nature chooses $t \in T$, and each informed agent $i$ of type $j$ knows her own $t_{j}$. Hence, conditional probabilities will be important: for each agent $i$ of type $j$ and every $t_{j} \in T_{j}$, the conditional probability of $t_{-j} \in T_{-j}$, given $t_{j}$ is denoted $q\left(t_{-j} \mid t_{j}\right)$.

Given an economy, let a coalition be a nonempty set of agents. Abusing notation slightly and making the simplifying convention that $T_{j} \times T_{j}=T_{j}$, for coalition $S$, we can denote $T_{S}=\prod_{j \in S} T_{j}$ and $T_{-S}=\prod_{j \notin S} T_{j}$. A feasible deterministic (state contingent) $S$-allocation, $x: T \mapsto R^{l s}$ (where $s$ denotes the cardinality of $S$ ), consists of a commodity bundle for each consumer in $S$ in each state such that $\sum_{i \in S} x_{i}(t) \leq \sum_{i \in S} e_{i}$ for all $t \in T$, and satisfying that $x\left(t_{S}, t_{-S}^{\prime}\right)=x\left(t_{S}, t_{-S}^{\prime \prime}\right)$ for all $t_{S} \in T_{S}$ and for all $t_{-S}^{\prime}, t_{-S}^{\prime \prime} \in T_{-S}$. (The latter assumption is made to exclude basic externalities across coalitions, i.e., the set of feasible allocations to a coalition is independent of the information held by the complement, although this may affect the utilities of agents in the coalition). We will denote by $\mathcal{A}_{S}$ the set of feasible deterministic state contingent allocations of $S$. With confusion being avoided by the context, we shall also use $\mathcal{A}_{S}$ to denote the set of feasible deterministic allocations in a given state: $\mathcal{A}_{S}=\left\{\left(x_{i}\right) \in \prod_{i \in S} X_{i} \mid \sum_{i \in S} x_{i} \leq \sum_{i \in S} e_{i}\right\}$. Similarly, recalling that $\mathbf{N}$ denotes the set of all agents in the economy, deterministic state contingent $\mathbf{N}$-allocations are simply referred to as deterministic allocations and the set of such deterministic allocations is denoted by $\mathcal{A}_{\mathbf{N}}$.

In this paper we assume that coalitions are formed at the interim stage, i.e., after each agent has received her private information. If coalitions are formed at the interim stage, then we need to specify the information that agents in a coalition are allowed to use in constructing an objection. Depending on the extent of such communication within the coalition, several interim core concepts have been proposed so far; see Forges, Minelli and 
Vohra (2002) or Forges and Serrano (2013) for surveys. This paper focuses specifically on the interim cores with endogenous information transmission, namely, the core with respect to (w.r.t.) equilibrium blocking, as explained next.

We shall work in environments in which the information state will not be verifiable, not even at the ex post stage. Thus, it becomes necessary to impose the incentive compatibility constraints into the analysis. If agent $i$ of type $j$ has private information $t_{j}$ and misrepresents it by reporting $t_{j}^{\prime}$ (while all other agents are truthful), she gets interim utility:

$$
U_{i}\left(x, t_{j}^{\prime} \mid t_{j}\right)=\sum_{t_{-j} \in T_{-j}} q\left(t_{-j} \mid t_{j}\right) u_{i}\left(x_{i}\left(t_{-j}, t_{j}^{\prime}\right),\left(t_{-j}, t_{j}\right)\right) .
$$

An allocation $x$ is incentive compatible (IC) if for every $i \in \mathbf{N}$, for every type $j$, and for every $t_{j} \in T_{j}$,

$$
U_{i}\left(x \mid t_{j}\right) \geq U_{i}\left(x, t_{j}^{\prime} \mid t_{j}\right)
$$

for every $t_{j}^{\prime} \in T_{j} \backslash\left\{t_{j}\right\}$. We denote the set of IC allocations by $\mathcal{A}_{\mathbf{N}}^{*}$.

Information transmission concerns ruling out some states as impossible, through the identification of smaller events. For an event $E \subseteq T$ and $t_{j} \in T_{j}$, let

$$
E_{-j}\left(t_{j}\right)=\left\{t_{-j} \in T_{-j} \mid\left(t_{j}, t_{-j}\right) \in E\right\}
$$

and

$$
E_{j}=\left\{t_{j} \in T_{j} \mid E_{-j}\left(t_{j}\right) \neq \emptyset\right\} .
$$

Consider an allocation rule $x \in \mathcal{A}_{\mathbf{N}}$, agent $i$ of type $j$ and information $t_{j}$, and an event $E$. Suppose $q\left(E_{-j}\left(t_{j}\right)\right)>0$. Then the interim utility conditional on $E$ can be expressed as:

$$
U_{i}\left(x \mid t_{j}, E\right)=\sum_{t_{-j} \in E_{-j}\left(t_{j}\right)} \frac{q\left(t_{-j} \mid t_{j}\right)}{q\left(E_{-j}\left(t_{j}\right) \mid t_{j}\right)} u_{i}\left(x_{i}\left(t_{-j}, t_{j}\right),\left(t_{-j}, t_{j}\right)\right) .
$$


The corresponding interim utility (conditional on $E$ ) if information $t_{j}$ is falsely reported as $t_{j}^{\prime}$ by agent $i$ of type $j$, while the others are truth-telling, is:

$$
U_{i}\left(x, t_{j}^{\prime} \mid t_{j}, E\right)=\sum_{t_{-j} \in E_{-j}\left(t_{j}\right)} \frac{q\left(t_{-j} \mid t_{j}\right)}{q\left(E_{-j}\left(t_{j}\right) \mid t_{j}\right)} u_{i}\left(x_{i}\left(t_{-j}, t_{j}^{\prime}\right),\left(t_{-j, t_{j}}\right)\right) .
$$

Given $E \subseteq T$, an $S$-allocation $x \in \mathcal{A}_{S}$ is $I C$ over $E$ if for every $i \in S$ for every type $j$, and for every $t_{j}, t_{j}^{\prime} \in E_{j}$ :

$$
U_{i}\left(x \mid t_{j}, E\right) \geq U_{i}\left(x, t_{j}^{\prime} \mid t_{j}, E\right) .
$$

The credible core, proposed in Dutta and Vohra (2005), is the set of incentive compatible allocations immune to credible objections. A coalition has a credible objection if it can identify an informational event such that the $t_{j}$ 's involved in the event are the only ones that prefer the alternative proposed to the status quo, given that the others behave as prescribed in the objection. This self-selection ensures that an agent with a given $t_{j}$ wishes to participate in the objection if and only if it is consistent with the objection's event. Therefore, the information transmitted in the objection via the event is "credible" in that those agents with $t_{j}$ 's that are not part of it have no incentive to join the objection.

Formally, a coalition $S$ has a credible objection if there exists $y^{\prime} \in \mathcal{A}_{S}$, and $E_{j} \subseteq T_{j}$ for type $j$ for all $i \in S$, where $q(E)$ is used for the product event $E=\prod_{j \in S} E_{j} \times T_{-S}$, such that:

(i) $U_{i}\left(y^{\prime} \mid t_{j}, E\right) \geq U_{i}\left(x \mid t_{j}, E\right)$ for all $i \in S$ and all $t_{j} \in E_{j}$, with strict inequality for some $i$ and $t_{j}$.

(ii) $U_{i}\left(y^{\prime} \mid t_{j}, E\right) \leq U_{i}\left(x \mid t_{j}, E\right)$ for all $i \in S$ and all $t_{j} \notin E_{j}$.

(iii) $y^{\prime} \in \mathcal{A}_{S}^{*}(E)$.

Condition (i) means that there is a product event over which all members of the coalition gain and (ii) implies that it is reasonable for them to believe 
this event since those $t_{j}$ 's who do not belong to this event would not join the alternative mechanism. Condition (iii) is simply physical feasibility of the objection.

To potentially destabilize a status quo, Myerson (2007) and Serrano and Vohra (2007) expand coalitional interactions and information transmission to also consider random plans, leading to the virtual utility core and the randomized mediated core, respectively. A random coalitional plan $\mu$ consists of a collection of probability distributions over feasible allocation rules for various coalitions. In particular, $\mu\left(S, y^{S}, t\right)$, where $y^{S} \in \mathcal{A}_{S}$, denotes the probability with which coalition $S$ is receiving $y^{S} \in \mathcal{A}_{S}$ when the (reported) state is $t \in T$. (We omit the formal definitions of these cores; the reader is referred to the original papers.)

Serrano and Vohra (2007) formalize coalitional stability by means of equilibria of voting games in which agents choose between the status-quo allocation and another feasible alternative. They answer the question of how much information gets transmitted within a coalition by requiring that the event that the objection uses coincides with the event over which an equilibrium rejection of the status-quo takes place for some Bayesian equilibrium of some communication mechanism used by the coalition. They also show that the above cores can be represented as a function of the class of voting mechanisms one allows, as summarized below. This gives rise to what we refer to as the core w.r.t. equilibrium blocking.

An incentive compatible allocation $x$ is in the core w.r.t. equilibrium blocking of some class of random communication mechanisms whenever there does not exist an equilibrium rejection of $x$, i.e., a random blocking plan against the status-quo $x$, for a communication mechanism in that class.

Particular cases of this definition are the following: 
- The credible core (Dutta and Vohra, 2005) turns out to be the core w.r.t. equilibrium blocking of single-coalition deterministic mechanisms.

- The virtual utility core (Myerson, 2007) turns out to be the core w.r.t. equilibrium blocking of measurable random mechanisms.

- The randomized mediated core (Serrano and Vohra, 2007) turns out to be the core w.r.t. equilibrium blocking of unrestricted random mechanisms.

All these notions are interim cores with endogenous information transmission: updated interim utilities are used to evaluate allocations after credible information transmission. It is known that any such core w.r.t. equilibrium blocking is included in the incentive compatible coarse core, defined in Vohra (1999). In general, the incentive compatible coarse core might be empty, even in quasilinear exchange economies, as shown in Forges, Mertens and Vohra (2002). Our task in this paper is to find sufficient conditions under which even the smallest core w.r.t. equilibrium blocking be nonempty in quasilinear economies.

Coalition $S$ is said to have an ex post objection to $x \in \mathcal{A}_{N}$ if there exists $y^{S} \in \mathcal{A}_{S}$ and $t \in T$ such that $u_{i}\left(y^{S}, t\right) \geq u_{i}(x, t)$ for all $i \in S$, with strict inequality for some $i \in S$. The ex post core is the set of all allocations to which no coalition has an ex post objection. It is the set of allocations that correspond to the classical core of the (complete information) ex post economy for all $t \in T$. Conditions under which the ex post core is nonempty are well-known. In particular, it is nonempty if preferences are continuous, monotonic and convex. 
The inclusion of the ex post core allocations satisfying IC in the core w.r.t. equilibrium blocking of unrestricted random communication mechanisms was shown in Kamishiro and Serrano (2011, Proposition 1), which follows an argument in Dutta and Vohra (2005) for the IC coarse core. For convenience, we restate it here as a proposition.

Proposition 1 In quasilinear exchange economies, all the ex post core allocations satisfying IC are contained in any core w.r.t. equilibrium blocking.

As argued in the beginning of this section, incentive problems become absent when multiple copies of each agent are present. Hence, we can easily obtain the following simple proposition.

Proposition 2 For all profiles $f$ with $f(j) \geq 2$ for all $j \in I$, any interim core w.r.t. equilibrium blocking is nonempty if agents' utilities are quasilinear and satisfy the standard classical assumptions.

Proof: Since $f(j) \geq 2$ for all $j \in I$, all the incentive constraints can be dropped (recall the opening paragraph of section 2). Therefore, all ex post allocations satisfy IC, and by Proposition 1, the ex post core, which is nonempty given the classical standard assumptions, is included in any interim core w.r.t. equilibrium blocking.

Our concern in this paper is dealing with scenarios in which incentive constraints matter. Then, one will have difficulties establishing nonemptiness of exact cores. We resort to approximate cores as a solution to the nonemptiness problem.

\subsection{Small Group Effectiveness and Approximate Cores}

The next property of small group effectiveness (SGE) is not defined for a single economy. The definition applies to all possible economies derived from 
a given pre-economy, without a specification of a fixed set of agents.

Definition. A pre-economy $\left(N, I,\left(T_{j}, u_{j}\right)_{j=1, \ldots, n}\right)$ satisfies small group effectiveness (SGE) in state $t$ if the derived pre-game $(N, \Psi)$ satisfies small group effectiveness; that is, given any real number $\varepsilon>0$, there is an integer $\eta_{0}(\varepsilon)$ such that for each profile $g$ on $N$, for some partition of $g$ into sub-profiles, say $\left\{g^{a}: a=1, \ldots, A\right\}$, where $\left\|g^{a}\right\| \leq \eta_{0}(\varepsilon)$ it holds that

$$
\Psi(g, t)-\sum_{a} \Psi\left(g^{a}, t\right)<\varepsilon_{0}\|g\|
$$

Intuitively, SGE ensures that "scarce types" of agents - those that appear in vanishingly small proportions as economies grow large - cannot have major impact on economic aggregates. SGE implies boundedness of the function $\frac{\Psi(g, t)}{\|g\|}$, per capita boundedness, and if scarce types are ruled out, then SGE is equivalent to simply boundedness of per capita payoffs (see Wooders (1994, Theorem 4).

An $\varepsilon$-approximate core requires that no group of agents can improve upon their allocation by more than $\varepsilon$ per capita. We might think of $\varepsilon$ as coalition formation and/or communication costs. ${ }^{6}$ For such an interpretation, think of an allocation as given and ask the question: Can any group of agents improve on the allocation after paying costs of communicating with other agents in the population?

Recall that an allocation is in the core of a game (derived from an economy with quasilinear utilities) if and only if there is an equal-treatment allocation in the core. Thus, given a state of the world $t$, a profile $f$ and the associated worth $\Psi(g, t)$ and $\varepsilon>0$, a (feasible) equal-treatment allocation $\left(x_{j}, j=\right.$ $1, \ldots, n)$ is in the $\varepsilon$-core if and only if for every sub-profile $g \leq f$ it holds

\footnotetext{
${ }^{6}$ The $\varepsilon$-core was introduced by Shapley and Shubik (1966).
} 
that

$$
\Psi(g, t) \leq \max \sum_{j} g(j) u_{j}\left(x_{j}, t\right)+\varepsilon\|g\| .
$$

From an argument identical to that in Proposition 1, it suffices to prove nonemptiness of the approximate ex post core with incentive compatibility, in order to show nonemptiness of any approximate core w.r.t. equilibrium blocking. We turn to such a result next.

\section{The Main Result: Monopolists of Scarce Information}

We analyze sequences of economies featuring monopolists of scarce information. That is, these are sequences in which $f(j)=1$ for at least one type $j \in I$ and where the number of uninformed agents grows large. For all such economies, we can show nonemptiness of any approximate interim core w.r.t. equilibrium blocking. The key argument is shown in sequences where $f(j)=1$ for all $j \in I$, but one can easily see how the same argument would apply to the other sequences (by treating types with $f(j) \geq 2$ as uninformed agents). Thus, we proceed to state and prove the following:

Theorem 3 Suppose that a quasilinear exchange pre-economy satisfies small group effectiveness in each state $t \in T$. Then, for every $\varepsilon>0$, there exists a number $M$ such that: For all profiles $f$ with $\|f\|>M$ and $f(j)=1$ for all $j \in I$, any $\varepsilon$-interim core w.r.t. equilibrium blocking is nonempty.

Proof: The proof is a combination of Proposition 3, which follows suit, and the inclusion of the ex post core with incentive compatibility in any core w.r.t. equilibrium blocking (Proposition 1). 
Proposition 4 Suppose that a quasilinear exchange pre-economy satisfies small group effectiveness in each state $t \in T$. Then for every $\varepsilon>0$, there exists a number $M$ such that: For all profiles $f$ with $\|f\|>M$ and $f(j)=1$ for all $j \in I$, the approximate $\varepsilon$ ex post core (with incentive compatibility) is nonempty.

Remark: In subsection 3.1, we provide sufficient conditions on the primitives of the pre-economy to ensure SGE.

Proof. To obtain a contradiction, let $\varepsilon_{0}>0$ be a number with the property that for each $n$ there is a profile $f^{n},\left\|f^{n}\right\|>n$, such that the economy with profile $f^{n}$ has an empty $3 \varepsilon_{0}$-core. We can assume that $\frac{f^{n}}{\left\|f^{n}\right\|} \rightarrow f$ for some function $f$ (by restricting attention to a subsequence if necessary).

From SGE in state $t \in T$, there is a bound $B$ such that for any $t \in T$ and any profile $g$ on $N$, for some partition of $g$ into sub-profiles (which could be state-dependent), say $\left\{g^{a}: a=1, \ldots, A\right\}$ where $\left\|g^{a}\right\| \leq B$ for each $g^{a}$, we have

$$
\Psi(g, t)-\sum_{a} \Psi\left(g^{a}, t\right)<\varepsilon_{0}\|g\|
$$

Let $M:=\max \left\{\left\{\max _{\|g\| \leq B, t \in T} \Psi(g, t)+2 B \varepsilon_{0}\right\}, 3 \varepsilon_{0}\right\}$.

In order to prove the proposition, we use a Lemma in Wooders (1992).

Lemma. Let $\left\{f^{m}\right\}$ be a sequence of profiles on $N$ such that $\left\|f^{m}\right\| \rightarrow \infty$ as $m \rightarrow \infty$ and $\left(1 /\left\|f^{m}\right\|\right) f^{m} \rightarrow f$ for some function $f$. Then, given any $\varepsilon$ there is a profile $h$ and an integer $m(\varepsilon)$ such that for each $m \geq m(\varepsilon)$, for some integer $r_{m}$ and some profile $l^{m}$ we have $r_{m} h+l^{m}=f^{m}$ and $\frac{\left\|l^{m}\right\|}{\left\|f^{m}\right\|}<\varepsilon$.

From this lemma, there is an integer $n_{0}$ and a profile $h$ such that for each $n \geq n_{0}$, for some integer $r_{n}$ and some profile $l^{n}$ we have

$$
r_{n} h+l^{n}=f^{n} \quad \text { and } \quad \frac{\left\|l^{n}\right\|}{\left\|f^{n}\right\|}<\frac{\varepsilon_{0}}{2 M} .
$$


Since $\frac{r_{n}\|h\|}{\left\|f^{n}\right\|} \geq 1-\frac{\varepsilon_{0}}{2 M}$ and $M>2 \varepsilon_{0}$, there is an $n^{\prime} \geq n_{0}$ such that for all $n \geq n^{\prime}$,

$$
r_{n}\|h\| \varepsilon_{0}>\left\|l^{n}\right\| M \quad \text { and } \quad r_{n}>B
$$

We will use this fact to construct an approximate ex post core allocation rule.

We note that no agent who has some private information belongs to $h$.

Let us consider the following allocation rule, which we call $z$ : all of the agents keep their endowment of numeraire, and the allocations of nonnumeraire goods are determined as follows:

- $e_{j}$, no matter which the state is, (if $j$ is not in the support of $h$ )

- $z_{j}^{*}(t)$, where $z_{j}^{*}(t)$ is an ex post Walrasian allocation (in state $t$ ) for agent $j$ in the economy consisting of the agent set $h$ (if $j$ is in the support of $h)$.

We note this allocation rule satisfies both resource constraints and incentive compatibility (if the agents keep their endowment of numeraire), because this allocation rule is state-independent for the informed agents.

Let $\hat{z}$ be the allocation rule obtained by making a transfer of numeraire from $z$ such that:

- each agent $i$ in $h$ gives up $\varepsilon_{0}$ units of numeraire, and

- each agent $i$ not in $h$ receives $M$ units of numeraire.

Since $r_{n}\|h\| \varepsilon_{0}>\left\|l^{n}\right\| M$, the above transfer of numeraire is feasible.

We now show that $\hat{z}$ is in the $2 \varepsilon_{0}$-core of the economy in each state. Suppose not. Then for some state $t \in T$ and some coalition $S \subset N$, letting $s$ denote the profile of $S$, we have

$$
\Psi(s, t)-\sum_{i \in S} u_{i}\left(\hat{z}_{i}(t), t\right)>2 \varepsilon_{0}\|s\|
$$


From SGE in state $t$, for some partition of $S$ into groups $\left\{S^{a}: a=\right.$ $1, \ldots, A\}$ with profiles $s^{1}, \ldots, s^{A}$ respectively and with $\left\|s^{a}\right\| \leq B$ for each $a$ we have

$$
\sum_{a} \Psi\left(s^{a}, t\right)-\sum_{i \in S} u_{i}\left(\hat{z}_{i}(t), t\right)+\varepsilon_{0}\|s\| \geq \Psi(s, t)-\sum_{i \in S} u_{i}\left(\hat{z}_{i}(t), t\right)>2 \varepsilon_{0}\|s\| .
$$

This implies that for some $a^{\prime}$,

$$
\Psi\left(s^{a^{\prime}}, t\right)-\sum_{i \in S^{a^{\prime}}} u_{i}\left(\hat{z}_{i}(t), t\right)>\varepsilon_{0}\left\|s^{a^{\prime}}\right\| .
$$

We cannot have the support of $s^{a^{\prime}}$ contained in the support of $r_{n} h$; otherwise we would have a contradiction to the fact that $z^{*}$ is in the core of any derived economy with profile of the total player set equal to $r_{n} h$ for all $n$. Therefore, for at least one $i \in S^{a^{\prime}}$, we must have $u_{i}\left(\hat{z}_{i}(t), t\right) \geq M$. But then

$$
\Psi\left(s^{a^{\prime}}, t\right)-\sum_{i \in S^{a^{\prime}}} u_{i}\left(\hat{z}_{i}(t), t\right)<\Psi\left(s^{a^{\prime}}, t\right)-M+2 \varepsilon_{0}(B-1)<0,
$$

since $\Psi\left(s^{a^{\prime}}, t\right)+2 \varepsilon_{0} B \leq M$, which gives us a contradiction.

\subsection{Conditions for SGE}

In this subsection we drop the notation $t$ for simplicity, but it should be understood that the analysis concerns an arbitrary ex post state $t$. We begin by providing a condition that is equivalent to SGE (Wooders, 2010, Theorem 1):

Definition (Small group negligibility, SGN) A pre-economy satisfies small group negligibility (SGN) if it satisfies boundedness of average (per capita) utility and if, for any sequence of profiles $\left\{f^{m}\right\}$ where $\left\|f^{m}\right\| \rightarrow \infty$ and $f^{m}(j) \neq 0$ if and only if $f^{m^{\prime}}(j) \neq 0$ for all $m^{\prime}, \frac{f^{m}}{\left\|f^{m}\right\|}$ exists and

$$
\lim _{m \rightarrow \infty} \frac{\Psi\left(f^{m}\right)}{\left\|f^{m}\right\|} \text { exists, }
$$


then for any sequence of profiles $g^{m}$ such that $\lim _{m \rightarrow \infty} \frac{f^{m}+g^{m}}{\left\|f^{m}+g^{m}\right\|}=\lim _{m \rightarrow \infty} \frac{f^{m}}{\left\|f^{m}\right\|}$ it holds that

$$
\lim _{m \rightarrow \infty} \frac{\Psi\left(f^{m}+g^{m}\right)}{\left\|f^{m}+g^{m}\right\|} \text { exists and equals } \lim _{m \rightarrow \infty} \frac{\Psi\left(f^{m}\right)}{\left\|f^{m}\right\|} .
$$

Proposition 5 A pre-economy with strictly concave utility functions in which the marginal utilities of nonnumeraire goods go to zero as consumption of any commodity goes to infinity satisfies small group effectiveness.

Proof. First, note that strict concavity of utility functions implies positive 'marginal utilities' for each non-numeraire commodity. Since we do not assume differentiability this statement requires some formalization. Strict concavity implies that for each agent type $j$ it holds that, given a sequence of non-numeraire commodity bundles $x_{j}^{-L m}$ with the property that, for some $\ell$, for all $k \neq \ell$ it holds that $x_{k j}^{-L m}=x_{k j}^{-L m^{\prime}}$ and $x_{\ell j}^{-L m} \rightarrow \infty$ as $m \rightarrow \infty$, it holds that

$$
\lim _{m \rightarrow \infty}\left|v_{j}\left(x_{j}^{-L m}+\mathbf{1}_{\ell j}\right)-v_{j}\left(x_{j}^{-L m}\right)\right| \rightarrow 0 .
$$

where $\mathbf{1}_{\ell j} \in \mathbb{R}^{L}$ denotes the vector with the component in the the $\ell^{t h}$ position equal to 1 and alll other components equal to zero..This observation will play a role in our proof.

Our method of proof is to apply Berge's Theorem of the Maximum to show that $\frac{\Psi(f)}{\|f\|}$ is a uniformly continuous function of $\frac{f}{\|f\|}$, which, in view of the equivalence of SGE and SGN, yields the result.

First, we need to set up the problem to apply Berge's Theorem. Let $x \in \mathbb{R}^{L}$. Define

$$
\mathcal{F}=\left\{\frac{1}{\|f\|} f \in \Delta^{n-1}\right\}
$$


Note that since utility functions are strictly concave, maximizing the sum of utilities of an economy with profile $f$ is equivalent to maximizing the sum of utilities over the set of allocations of commodities that treat all agents of the same type equally. Thus, given $f$, we can maximize the following weighted sum of utilities:

$$
h(x, f)=\sum_{j} \frac{f(j)}{\|f\|} v_{j}\left(x_{j}^{-L}\right)
$$

over the set of allocations $x$ such that

$$
\sum_{j} \frac{f(j)}{\|f\|} x_{j}^{-L}=\sum_{j} \frac{f(j)}{\|f\|} e_{j}^{-L}
$$

where $x_{j}^{-L}$ is the allocation to each agent of type $j$. (Note that $h$ is the function $\Psi$ but with the allocation of the numeraire commodity taken into account. The numberaire can be allocated arbitrarily without affecting the maximal sum of utilities.)

From strict concavity of the functions $v_{j}$ it follows that that $h$ is strictly concave and continuous. Define the correspondence $\bar{C}$ mapping $\mathcal{F}$ into $\mathbb{R}_{+}^{-L n}$ by

$$
\bar{C}(f)=\left\{x \in R_{+}^{-L n}: \sum_{j} \frac{f(j)}{\|f\|} x_{j}^{-L}=\sum_{j} \frac{f(j)}{\|f\|} e_{j}^{-L}\right\} .
$$

Note that $\bar{C}$ is a continuous correspondence on $\mathcal{F}$.

Note also that $\bar{C}(f)$ is not a compact set since, for example, all agents of one type may be assigned the total endowment of the economy. The following Lemma shows that, without loss of generality, we can restrict $\bar{C}$ to a compact set.

Lemma. There exists a constant $K$ such that, for any profile $f$, if $x \in \bar{C}(f)$ and $x$ maximizes

$$
h(x, f)=\sum_{j} \frac{f(j)}{\|f\|} v_{j}\left(x_{j}^{-L}\right)
$$


then $x \leq K \mathbf{1}_{n}$.

Proof of the Lemma. Suppose that the statement of the lemma is false. Then there exists a sequence of profiles $f^{m}$ such that $\left\|f^{m}\right\| \rightarrow \infty$ and a sequence of allocations of non-numeraire commodities $x^{m} \in \bar{C}(f)$ such that, for some component $j \in N$ and some $\ell \in\{1, \ldots, L-1\}$, it holds that $x_{\ell j}^{m}>m$ for each $m$. Note that since $x_{\ell j}^{m}$ goes to infinity as $m$ becomes large, it holds that the 'marginal utility' of good $\ell$ for type $j$ goes to zero. That is, given any $\lambda>0$ there is a $m_{0}$ such that for all $m>m_{0}$

$$
u_{j}\left(x_{\ell j}^{m}\right)-u_{j}\left(x_{\ell j}^{m}-\mathbf{1}_{\ell}\right)<\lambda .
$$

Since utility functions for non-numeraire commodities are strictly concave the maximum of total utility is achieved with equal marginal utilities for all agents that consume a commodity. Under the assumption that all agents have positive marginal utility for all commodities, for sufficently small $\lambda$ it must hold that for all types

$$
u_{k}\left(x_{\ell k}^{m}\right)-u_{j}\left(x_{\ell k}^{m}-\mathbf{1}_{\ell}\right)<\lambda .
$$

This is impossible since it requires that for all agent types $k$ it $x_{\ell k}^{m}$ goes to infinity as $m$ grows large. Since endowments $e_{j}$ are fixed, the average allocation of each commodity is bounded and thus the average cannot go to infinity.

Let $\boldsymbol{C}$ be a compact set such that if $x \in \bar{C}(f)$ and $x$ maximizes $h(x, f)=$ $\sum_{j} \frac{f(j)}{\|f\|} u_{j}\left(x_{j}^{-l}\right)$ then $x \in \mathbf{C}$ and for each profile $f$ let $C(f)=\bar{C}(f) \cap \mathbf{C}$.

For $x \in R^{-L n}$ and $\frac{f}{\|f\|} \in \mathcal{F}$ let

$$
h^{*}\left(\frac{f}{\|f\|}\right)=\max \left\{h\left(x, \frac{f}{\|f\|}\right): x \in C(f)\right\}
$$


and (although we do not need it),

$$
C^{*}(f)=\arg \max \left\{h\left(x, \frac{f}{\|f\|}\right): x \in C(f)\right\} .
$$

Since $C$ is continuous on its entire domain, from Berge's Theorem it follows that so is $h^{*}$, and that $C^{*}\left(\frac{f}{\|f\|}\right)$ is nonempty, compact-valued, and continuous (which is somewhat stronger than the conclusion of Berge's Theorem).

Let

$$
\frac{\Psi(f)}{\|f\|}=h^{*}\left(\frac{f}{\|f\|}\right)+\frac{\sum_{j} f(j) e_{j}^{l}}{\|f\|}
$$

. Since $h^{*}$ is uniformly continuous, it follows that SGN is satisfied.

\subsection{Relaxing the Assumptions}

It is important to understand how much one can relax the assumptions under which our results obtain. We discuss relaxations of the informational assumptions in our next section, while here we concentrate on the assumptions made on utility functions.

If we relax only the assumption about the condition on the marginal utilities, then we can already find an example such that the approximate ex post core is empty. (The following example also drops strict concavity, but as we explain at the end of this section, we can also find an example satisfying strict concavity by making a slight variant thereof. We omit the details of that somewhat more involved example, for expositional reasons.)

Example. There are two goods $x$ and $y$ ( $y$ is numeraire) and two agents in the original economy. Suppose there are two states $s$ and $t$. Agent 1 knows the true state as his own type while agent 2 is uninformed. Let $e_{1}=(1,2)$ and $e_{2}=(2,1)$. The utility functions are as follows:

$$
\begin{array}{ll}
u_{1}(x, y, s)=1.5 x+y, & u_{2}(x, y, s)=x+y \\
u_{1}(x, y, t)=2 x+y, & u_{2}(x, y, t)=2.5 x+y
\end{array}
$$


This economy violates SGE in both states. We provide the detailed computations for state $s$ (the calculations for state $t$ are similar). In state $s$, consider a profile with a single copy of agent 1 , and $n_{2}$ copies of agent 2 , where $n_{2}$ is sufficiently large. Then,

$$
\Psi\left(0, n_{2}\right)=3 n_{2}, \quad \Psi(1,0)=3.5,
$$

$\Psi\left(1, n_{2}\right)=1.5\left(1+2 n_{2}\right)+\left(1+n_{2}\right)=2.5+4 n_{2}$,

Therefore, $\frac{\Psi\left(1, n_{2}\right)-\Psi(1,0)-\Psi\left(0, n_{2}\right)}{1+n_{2}}=$ approximately equal to 1 , which stays away from zero, and hence SGE is not satisfied.

That is, in an economy with many agents of type 2 and only one agent of type 1 , in state $s$, whether the type 1 agent belongs to a coalition can have a very large effect on the payoff to that coalition.

\section{Proposition.}

Consider an economy where there is only one agent 1 and $M$ copies of agent 2. Let $\varepsilon<\frac{1}{4}$. Then for all $M$, the ex post $\varepsilon$-core with (interim) incentive compatibility is empty.

Proof.

Let $\left(x_{1}(\cdot), y_{1}(\cdot)\right),\left(x_{2, k}(\cdot), y_{2, k}(\cdot)\right)(k=1,2, \ldots, M)$ be an allocation rule that belongs to the ex post $\varepsilon$-core with (interim) incentive compatibility.

(1) By the $\varepsilon$-efficiency of the allocation in state $s$,

$$
1.5 x_{1}(s)+\sum_{k} x_{2, k}(s) \geq 1.5(1+2 M)-(1+M) \varepsilon .
$$

The resource constraint of good $x$ in state $s$ is $x_{1}(s)+\sum_{k} x_{2, k}(s) \leq 1+2 M$.

Combining these two inequalities,

$$
1.5 x_{1}(s)+\left(1+2 M-x_{1}(s)\right) \geq 1.5(1+2 M)-(1+M) \varepsilon .
$$

Therefore, $x_{1}(s) \geq 1+2 M-2(1+M) \varepsilon$. 
(2) By incentive compatibility in each state,

State $s: 1.5 x_{1}(s)+y_{1}(s) \geq 1.5 x_{1}(t)+y_{1}(t)$.

State $t: 2 x_{1}(t)+y_{1}(t) \geq 2 x_{1}(s)+y_{1}(s)$.

Combining these two, $1.5 x_{1}(s)+y_{1}(s)+0.5 x_{1}(t) \geq 2 x_{1}(t)+y_{1}(t) \geq$ $2 x_{1}(s)+y_{1}(s)$.

Hence, $x_{1}(t) \geq x_{1}(s)$.

(3) The resource constraint of good $x$ in state $t$ is:

$$
\begin{aligned}
\sum_{k} x_{2, k}(t) & \leq 1+2 M-x_{1}(t) \\
& \leq 1+2 M-x_{1}(s) \\
& \leq 1+2 M-\{1+2 M-2(1+M) \varepsilon\}=2(1+M) \varepsilon .
\end{aligned}
$$

(4) By $\varepsilon$-efficiency in state $t$,

$$
2 x_{1}(t)+2.5 \sum_{k} x_{2, k}(t) \geq 2.5(1+2 M)-(1+M) \varepsilon .
$$

The resource constraint of good $x$ in state $t$ is $x_{1}(t)+\sum_{k} x_{2, k}(t) \leq 1+2 M$. Combining these two,

$$
\begin{aligned}
2.5 \sum_{k} x_{2, k}(t) & \geq 2.5(1+2 M)-(1+M) \varepsilon-2 x_{1}(t) \\
& \geq 2.5(1+2 M)-(1+M) \varepsilon-2\left(1+2 M-\sum_{k} x_{2, k}(t)\right) \\
& =0.5+M-(1+M) \varepsilon-2 \sum_{k} x_{2, k}(t) .
\end{aligned}
$$

Hence, $\sum_{k} x_{2, k}(t) \geq(1+M)-2(1+M) \varepsilon . \quad(* *)$

If an allocation rule were to satisfy both $(*)$ and $(* *)$, then

$2(1+M) \varepsilon \geq(1+M)-2(1+M) \varepsilon$, then it would hold that $\varepsilon \geq \frac{1}{4}$, a contradiction. 
Remark. Almost the same arguments can be applied even if the utility functions are as follows,

$$
\begin{array}{ll}
u_{1}(x, y, s)=f(x)+y, & u_{2}(x, y, s)=g(x)+y \\
u_{1}(x, y, t)=f(x)+0.5 x+y, & u_{2}(x, y, t)=h(x)+y
\end{array}
$$

where $f, g$, and $h$ are all strictly concave functions satisfying:

- $f^{\prime}(x)<2$ for all $x$ and $f$ is asymptotic to the straight line with slope of 1.5 (i.e., $f^{\prime}(x) \rightarrow 1.5$ as $x \rightarrow \infty$ ),

- $g^{\prime}(x)<1.5$ for all $x$, and

- $h^{\prime}(x) \rightarrow 2.5$ as $x \rightarrow \infty$.

As in the previous proof, steps 1 and 4 can be shown because in state $s$ the marginal utility of good $x$ for agent 1 is greater than the one of agent 2 for all amounts of $x$, while in state $t$ the marginal utility of good $x$ for agent 2 is greater than the one of agent 1 for all amounts of $x$.

\section{Informational Smallness in Signal-Based Pro- cesses}

So far this paper has analyzed situations in which the "total amount of information" is small, in the sense that the number of agents with private information is uniformly bounded, while the number of uninformed agents grows without bound.

As a different distribution of information, one can consider sequences of economies such that all agents are informationally small in the sense of McLean and Postlewaite (2002). As such, SGE holds in these settings, as 
there are no scarce types. ${ }^{7}$ These authors consider a model in which the agents' utility functions depend on an underlying but unobserved state of nature and in which each agent will receive a private signal that is correlated with the state of nature. Roughly speaking, agents are informationally small if, with high probability, agents are not able to change the estimated state by misreporting their signals. In this sequence we can also obtain a nonemptiness result of the approximate core for sufficiently large economies.

\subsection{Notation and definitions}

Since the model employed in this section is slightly different from that used in the rest of the paper, we begin with a few preliminaries in order to define a pre-economy and an economy. Let $\Theta=\left\{\theta_{1}, \ldots, \theta_{m}\right\}$ denote a (finite) state space and let $T_{1}, \ldots, T_{n}$ be finite sets, where $T_{j}$ represents the set of possible signals that agent $j \in N$ might receive. In this model, nature chooses an element $\theta \in \Theta$. The state of nature is unobservable but each agent $j$ receives a "signal" that is correlated with nature's choice of $\theta$. We denote the probability distribution on $\Theta \times T$ as $P$. We assume that for every $\theta, \theta^{\prime}$ with $\theta \neq \theta^{\prime}$, there exists a $t \in T$ such that $P(t \mid \theta) \neq P\left(t \mid \theta^{\prime}\right)$.

The consumption set of each agent $X_{j}$ is $R_{+}^{L-1} \times R$ and for each $\theta \in \Theta, e_{j} \in$ $X_{j}$ denotes the (state independent) endowment of agent $j$ in state $\theta \in \Theta$. The preferences of agent $j$ are given by a utility function $u_{j}: R_{+}^{L-1} \times R \times \Theta \rightarrow R$ where $u_{j}\left(x_{j}, \theta\right)=v_{j}^{-L}\left(x_{j}^{-L}, \theta\right)+x_{j}^{L}$ is the utility function of agent $j$ in state $\theta$. We note that in this model agents' utility functions do not depend on $T$. The collection $\mathcal{E}=\left(\left\{u_{j}, X_{j}, e_{j}, T_{j}\right\}_{j \in N}, \Theta, P\right)$ will be called a private information pre-economy. An allocation rule $x=\left(x_{1}, \ldots, x_{n}\right)$ for the private information

\footnotetext{
${ }^{7}$ That is, there are many close substitutes for each agent. As noted in the introduction if there are no agents of scarce types then SGE is equivalent to simply boundedness of per capita payoffs.
} 
pre-economy is a collection of functions $x_{j}: T \rightarrow \mathcal{A}_{N}$. For each state $\theta \in \Theta$, the collection $\left\{u_{j}(\cdot, \theta), e_{j}\right\}_{j \in N}$ defines an associated (complete information) pre-economy.

In this section, we confine our discussion to exact replicas; that is, we sequences of economies where the $r^{\text {th }}$ economy has $r$ agents of each type. This yields simpler mathematical expressions and a more transparent argument. ${ }^{8}$ That is, we consider only a profile $f$ such that there exists a number $M$ satisfying $f(j)=M$ for all $j$.

Given a pre-economy $\mathcal{E}$ and a profile $f$, let

$$
\mathcal{E}_{f}=\left(\left\{u_{(j, k)}, X_{(j, k)}, e_{(j, k)}, T_{(j, k)}\right\}_{j \in N, k=1, \ldots, M}, \Theta, P_{f}\right)
$$

be a private information economy with $\|f\|(=M \times n)$ agents satisfying the following conditions. (Hereafter we denote $T_{f}:=\prod_{j \in N, k=1, \ldots, M} T_{(j, k)}$.)

(1) $e_{(j, k)}=e_{j}$ and $X_{(j, k)}=X_{j}$ for all $j \in N$ and all $k=1, \ldots, M$,

(2) $u_{(j, k)}(x, \theta)=u_{j}(x, \theta)$ for all $x \in R_{+}^{l-1} \times R, j \in N$ and $k=1, \ldots, M$.

(3) $T_{(j, k)}=T_{j}$ for all $j \in N$ and $k=1, \ldots, M$.

(4) $P_{f}$ is a probability distribution on $\Theta \times T_{f}$ satisfying the following 9 :

(a) For each $k=1,2, \ldots, M$, and each $\left(\theta, t_{1}, \ldots, t_{n}\right) \in \Theta \times T$,

$$
P\left(\theta, t_{(1, k)}, t_{(2, k)}, \ldots, t_{(n, k)}\right)=P\left(\theta, t_{1}, t_{2}, \ldots, t_{n}\right)
$$

(b) for each $\theta$, the probability distributions over

$$
\left(T_{(1,1)}, T_{(2,1)}, \ldots, T_{(n, 1)}\right), \ldots,\left(T_{(1, M)}, T_{(2, M)}, \ldots, T_{(n, M)}\right)
$$

\footnotetext{
${ }^{8}$ Extending our results for the case of more general sequences is an open question; we explain some of its difficulties in the sequel. Note, however, that since agents of different types may be identical, the restiction to replica sequences is not as restrictive as it might first seems.

${ }^{9}$ These sequences are called conditionally independent replicas in McLean and Postlewaite (2002).
} 
are independent given $\theta$.

Thus, $\mathcal{E}_{f}$ is a private information economy with $\|f\|$ agents containing $M$ "copies" of each agent $j \in N$. It follows that, if $M$ is sufficiently large for all $j$, then one could assign a probability to each state that is arbitrarily close to 1 . We note that no agent's information is redundant in this process: regardless of the number of copies, each agent still has information that cannot be inferred from the aggregate information of other agents.

\subsection{Nonemptiness of the Approximate Core}

McLean and Postlewaite (2002) show that, if the economy is sufficiently replicated, all agents are informationally small: the conditional distribution on the state of nature does not vary much in that agent's signal if other agents' signals are known. Moreover, they construct a mechanism that is incentive compatible, ex post individually rational, and nearly ex post Pareto efficient.

Developing the result in McLean and Postlewaite (2002), if the original pre-economy is sufficiently replicated, we can construct an incentive compatible mechanism that is a bit different than the one considered by these authors in Step 3 of their proof of Theorem 2, in such a way that it allocates an ex post core allocation when their received signal cannot estimate the true state with high probability. That is, McLean and Postlewaite (2002) constructed an incentive compatible mechanism that satisfies (i)-(iii) in the lemma below (for large $r$ ). Their constructed mechanism allocates the endowments to the agents when $t^{r} \in T^{r} \backslash\left[\cup_{i=1}^{m} B_{i}\right]$. Therefore, their rule does not belong to an (approximate) ex post core in general. If one tries to modify their rule directly so that it allocates an ex post core allocation for $t^{r} \in T^{r} \backslash\left[\cup_{i=1}^{m} B_{i}\right]$, then a key inequality of their proof to show incentive compatibility breaks 
down. In order to overcome this problem, we present a variant of an argument in Kamishiro (2011), who takes a collection $B_{1}, \ldots, B_{m}$ of $T^{r}$ somewhat different from the one in McLean and Postlewaite (2002). By doing so, for sufficiently large $r$, one can find an incentive compatible rule so that it allocates an ex post core allocation when $t^{r} \in T^{r} \backslash\left[\cup_{i=1}^{m} B_{i}\right]$. (In order for the argument to go through, one needs to take $r$ larger than the one considered in McLean and Postlewaite (2002). To underscore the difference with Kamishiro (2011), we remark that that paper fixes the set of agents and assumes they have very accurate signals in order to establish nonemptiness of the exact coarse core, while we increase the number of agents and prove nonemptiness of approximate cores.) With such a change, a result similar to McLean and Postlewaite's Step 3 in the proof of Theorem 2 still holds (see also Lemma 4.5 in Kamishiro, 2011). This argument can be formally stated in the following lemma.

Lemma 6 For each $\eta>0$ and a feasible allocation for a pre-economy $\mathcal{A}=$ $\left(\zeta_{j}\left(\theta_{1}\right), \ldots, \zeta_{j}\left(\theta_{m}\right)\right)_{j \in N}$ in each state, there exists a number $\hat{M}$ such that: for all $M>\hat{M}$ and every profile $f$ satisfying $f(j)=M$ for all $j$ there exist an incentive compatible allocation $z(\cdot)$ for $\mathcal{E}_{f}$ and a collection $B_{1}, \ldots, B_{m}$ of disjoint subsets of $T^{f}$ such that

(i) $\operatorname{Pr}\left\{\tilde{t}^{f} \in \cup_{i=1}^{m} B_{i}\right\} \geq 1-\eta$,

(ii) $\operatorname{Pr}\left\{\tilde{\theta}=\theta_{i} \mid \tilde{t}^{f}=t^{f}\right\} \geq 1-\eta$ for all $i=1, \ldots, m$ and all $t^{f} \in B_{i}$,

(iii) for each agent $j$ in the profile $f$, all $i=1, \ldots, m$ and all $t^{f} \in B_{i}$,

$$
u_{j}\left(z_{j}\left(t^{f}\right) ; \theta_{i}\right) \geq u_{j}\left(\zeta_{j}\left(\theta_{i}\right), \theta_{i}\right)-\eta \text {. }
$$

(iv) for each agent $j$ in the profile $f$ and all $t^{f} \in T^{f} \backslash\left[\cup_{i=1}^{m} B_{i}\right]$,

$$
z_{j}\left(t^{f}\right)=\hat{x}_{j}\left(t^{f}\right)
$$


where $\left(\hat{x}_{j}\left(t^{f}\right)\right)_{j}$ is an ex post core allocation in type profile $t^{f}$ with agents' profile $f$.

(Here, $B_{i}$ can be interpreted as the set of type profile such that the true state is estimated as $\theta_{i}$ with almost probability one.)

The restriction to replicas can be explained with the following technical remark. The number $\hat{M}$ depends upon the allocation $\zeta$ which was defined for the pre-economy. In other words, we have to pick an allocation in each state before setting the number $\hat{M}$. This makes it difficult to argue the case of more general sequences, beyond replicas. Following the tecnhiques of the theory of cooperative games with many players and a compact metric space of player types, as in Wooders (2010), we conjecture that appropriate compactness of the space of agent types would allow the extension of our results, but this is beyond the scope of the current paper.

From this lemma, we can obtain the following nonemptiness result of the approximate core.

Theorem 7 For every $\varepsilon>0$, there exists a number $\hat{M}$ such that: for all $M>\hat{M}$ and every profile $f$ satisfying $f(j)=M$ for all $j$, any approximate $\varepsilon$ interim core w.r.t. equilibrium blocking of a private information economy $\mathcal{E}_{f}$ is nonempty.

Proof: Given $\varepsilon>0$, let

$$
K:=\max _{\theta} \max _{j} v_{j}^{-L}\left(\sum_{j=1}^{n} e_{j}^{-L}, \theta\right)+\sum_{j=1}^{n} e_{j}^{L} \quad \text { and } \quad \eta:=\frac{\varepsilon}{1+K} .
$$

We pick a Walrasian equilibrium allocation for all the economies in a replication sequence, which in the case of replicas is simply an allocation for the basic economy $\left(\zeta_{j}\left(\theta_{1}\right), \ldots, \zeta_{j}\left(\theta_{m}\right)\right)_{j \in N}$ with one agent of each type. Given 
$\eta$, we are able to choose $\hat{M}$ in accordance with Lemma 6 , such that for all $M>\hat{M}$ and every profile $f$ satisfying $f(j)=M$ for all $j$ there exist an incentive compatible allocation $z(\cdot)$ for $\mathcal{E}_{f}$ satisfying the conditions in Lemma 6.

It suffices to show that this allocation $z$ is not ex post $\varepsilon$-blocked by any coalition $S .^{10}$ If $t^{f} \in T^{f} \backslash\left[\cup_{k=i}^{m} B_{i}\right]$, then there is no such blocking from condition (iv) in Lemma 6 . So suppose that there exist $\left(y_{j}^{S}\right)_{j \in S} \in \mathcal{A}_{S}$ and $t^{f} \in B_{i}$ satisfying

$$
\sum_{j \in S} \sum_{\theta \in \Theta} u_{j}\left(y_{j}^{S}, \theta\right) P\left(\theta \mid t^{f}\right)>\sum_{j \in S} \sum_{\theta \in \Theta} u_{j}\left(z_{j}\left(t^{f}\right), \theta\right) P\left(\theta \mid t^{f}\right)+\varepsilon \cdot|S| .
$$

We will show that this leads to a contradiction.

For each $t^{f} \in B_{i}$,

$$
\begin{aligned}
\sum_{j \in S} \sum_{\theta \in \Theta} u_{j}\left(z_{j}\left(t^{f}\right), \theta\right) P\left(\theta \mid t^{f}\right) & \geq(1-\eta) \sum_{j \in S} u_{j}\left(z_{j}\left(t^{f}\right), \theta_{i}\right) \\
& \geq(1-\eta) \sum_{j \in S}\left[u_{j}\left(\zeta_{j}\left(\theta_{i}\right), \theta_{i}\right)-\eta\right] \\
& >(1-\eta) \sum_{j \in S} u_{j}\left(\zeta_{j}\left(\theta_{i}\right), \theta_{i}\right)-\eta \cdot|S|
\end{aligned}
$$

On the other hand,

$$
\sum_{j \in S} \sum_{\theta} u_{j}\left(y_{j}^{S}, \theta\right) P\left(\theta \mid t^{f}\right) \leq(1-\eta) \sum_{j \in S} u_{j}\left(y_{j}^{S}, \theta_{i}\right)+\eta \cdot K \cdot|S| \cdot
$$

\footnotetext{
${ }^{10}$ Our use of the term ex post in this section refers to events that occur after the realization of the signal profile $t$, but before the realization of the state $\theta$ (allocations can depend on agents' types but not on $\theta$, which is assumed to be unobservable).
} 
These imply that

$$
\begin{aligned}
(1-\eta) \sum_{j \in S} u_{j}\left(y_{j}^{S}, \theta_{i}\right)+\eta \cdot K \cdot|S| & \geq \sum_{j \in S} \sum_{\theta} u_{j}\left(y_{j}^{S}, \theta\right) P\left(\theta \mid t^{f}\right) \\
& >\sum_{j \in S} \sum_{\theta \in \Theta} u_{j}\left(z_{j}\left(t^{f}\right), \theta\right) P\left(\theta \mid t^{f}\right)+\varepsilon \cdot|S| \\
& >(1-\eta) \sum_{j \in S} u_{j}\left(\zeta_{j}\left(\theta_{i}\right), \theta_{i}\right)-\eta \cdot|S|+\varepsilon \cdot|S| .
\end{aligned}
$$

Thus we conclude that

$$
\sum_{j \in S} u_{j}\left(y_{j}^{S}, \theta_{i}\right)>\sum_{j \in S} u_{j}\left(\zeta_{j}\left(\theta_{i}\right), \theta_{i}\right)
$$

contradicting the assumption that $\left(\zeta_{j}\left(\theta_{i}\right)\right)_{j \in N}$ is a Walrasian allocation of the associated economy in state $\theta_{i}$, which therefore must belong to the core of the $M \times n$ agents' economy.

\section{Concluding Remarks}

This paper has offered a number of nonemptiness results for interim cores in sufficiently large quasilinear economies. When incentive constraints can be dropped, one can easily adapt arguments in previous literature to show nonemptiness of any exact interim core w.r.t. equilibrium blocking.

However, when incentive constraints still matter, either because of exclusive information or because of independent types in signal-based processes, the exact core may be empty. For such cases, we have shown how adapting the condition of small group effectiveness is useful in order to prove the nonemptiness of any approximate interim core w.r.t. equilibrium blocking in sequences of economies based on a pre-economy of types. The key argument, which is also applied in signal-based replica processes, relies on finding ex post core allocations that pass the interim incentive compatibility test. 
Finally, since the inclusion of the incentive compatible ex post core in the interim cores with endogenous communication holds for every quasilinear economy, once a sufficiently large economy is found with the approximate interim core being nonempty, any replica of such a large economy (considering different kinds of replicas, such as ex post, independent, or signal-based, as done in Kamishiro and Serrano, 2011) also preserves our nonemptiness results, thereby extending the classes of information structures that are compatible with nonempty approximate cores.

\section{References}

Dutta, B., Vohra, R., (2005). "Incomplete Information, Credibility and the Core," Mathematical Social Sciences 50, 148-165.

Forges, F., Serrano, R., (2013). "Cooperative Games with Incomplete Information: Some Open Problems," International Game Theory Review 15, 301-317.

Forges, F., Mertens, J.-F., Vohra, R., (2002). "The Ex Ante Incentive Compatible Core in the Absence of Wealth Effects," Econometrica 70, 18651892.

Forges, F., Minelli, E., Vohra, R., (2002). "Incentive and the Core of an Exchange Economy: a Survey," Journal of Mathematical Economics 38, 1-41.

Kamishiro, Y., (2011). "Informational Size and the Incentive Compatible Coarse Core in Quasilinear Economies," Games and Economic Behavior 71, 513-520.

Kamishiro, Y., Serrano, R. (2011). "Equilibrium Blocking in Large Quasilinear Economies," Mathematics of Operations Research 36, 552-567.

McLean, R. P., Postlewaite, A., (2002). "Informational Size and Incentive 
Compatibility," Econometrica 70, 2421-2453.

Myerson, R. B., (2007). "Virtual Utility and the Core for Games with Incomplete Information," Journal of Economic Theory 136, 260-285.

Serrano, R., Vohra, R., (2007). "Information Transmission in Coalitional Voting Games," Journal of Economic Theory 134, 117-137.

Serrano, R., Vohra, R., Volij, O., (2001). "On the Failure of Core Convergence in Economies with Asymmetric Information," Econometrica 69, 1685-1696.

Shapley, L. S., M. Shubik (1966), "Quasi-Cores in a Monetary Economy with Nonconvex Preferences," Econometrica 34, 805-827.

Vohra, R., (1999). "Incomplete Information, Incentive Compatibility and the Core," Journal of Economic Theory 86, 123-147.

Wilson, R., (1978). "Information, Efficiency and the Core of an Economy," Econometrica 46, 807-816.

Wooders, M., (1992). "Inessentiality of Large Groups and the Approximate Core Property; An Equivalence Theorem," Economic Theory 2, 129-147.

Wooders, M., (1994). "Equivalence of Games and Markets," Econometrica 62, 1141-1160.

Wooders, M., (2010). "Cores of Many-player Games; Nonemptiness and Equal Treatment," Review of Economic Design 14, 131-162. 